\title{
BMJ Open Parental attitudes and willingness to donate children's biospecimens for congenital heart disease research: a cross-sectional study in Shanghai, China
}

\author{
Hongxiang Gao, ${ }^{1}$ Jun Jiang, ${ }^{1}$ Bei Feng, ${ }^{1}$ Aihua Guo, ${ }^{2}$ Haifa Hong, ${ }^{2}$ Shijian Liu ${ }^{2}$
}

To cite: Gao H, Jiang J, Feng B, et al. Parental attitudes and willingness to donate children's biospecimens for congenital heart disease research: a cross-sectional study in Shanghai, China. BMJ Open 2018;8:e022290. doi:10.1136/ bmjopen-2018-022290

- Prepublication history for this paper is available online. To view these files, please visit the journal online (http://dx.doi. org/10.1136/bmjopen-2017022290).

$\mathrm{HG}$ and JJ contributed equally.

Received 14 March 2018 Revised 16 August 2018 Accepted 30 August 2018

Check for updates

(C) Author(s) (or their employer(s)) 2018. Re-use permitted under CC BY-NC. No commercial re-use. See rights and permissions. Published by BMJ.

${ }^{1}$ Department of Cardiothoracic Surgery, Shanghai Children's Medical Center, Shanghai Jiaotong University School of Medicine, Shanghai, China ${ }^{2}$ Department of Biobank, Pediatric Translational Medicine Institute, Shanghai Children's Medical Center, Shanghai Jiaotong University School of Medicine, Shanghai, China

\section{Correspondence to}

Dr Haifa Hong;

honghaifa@scmc.com.cn and Dr

Shijian Liu;

liushijian@scmc.com.cn

\section{ABSTRACT}

Objectives To assess attitudes and willingness of parents of children with congenital heart disease (CHD) regarding donating biospecimens for future CHD research, and to identify factors associated with biospecimen donation. Design Face-to-face cross-sectional survey data were analysed using logistic regression.

Setting Cardiothoracic Surgery Inpatient Department, Shanghai Children's Medical Centre.

Participants Parents of children attending the cardiothoracic surgery inpatient department at Shanghai Children's Medical Center, 1 March-31 December 2016.

Primary and secondary outcome measures Willingness and motivation regarding donating children's biospecimens, and ethical and legal considerations concerning children's future willingness to donate. Results of 550 parents, 508 completed the questionnaire (response rate $=92.4 \%)$. Overall, $69.1 \%(n=351)$ were willing to donate their children's biospecimens for medical research. Multivariate analysis indicated higher education level (college/graduate degree: OR 2.435, 95\% Cl 1.221 to 4.857, $\mathrm{p}=0.012$; high school: $\mathrm{OR} 1.827,95 \% \mathrm{Cl} 1.190$ to $2.804, p=0.006)$ and children's hospitalisation history (OR $1.581 ; 95 \% \mathrm{Cl} 1.069$ to $2.338, \mathrm{p}=0.022$ ) were positively associated with willingness to donate. The most common motivation for donation was potential benefit to other children with $\mathrm{CHD}(81.2 \%, \mathrm{n}=285)$. The main barriers to donation were physical discomfort to their children $(52.3 \%, n=54)$ and concerns about personal privacy $(47.1 \%, n=48)$. Most parents $(86.0 \%, n=302)$ wanted to be informed of research results using their children's donated biospecimens, and $34.8 \%(n=177)$ believed that children aged 10-18 years had the right to consent independently to research participation.

Conclusions Nearly $70 \%$ of the parents in this study were willing to donate their children's biospecimens for future CHD research. Parents' education level and children's hospitalisation history influenced willingness to donate. Most parents wanted to receive the research results related to their children's biospecimens.

\section{BACKGROUND}

Congenital heart disease (CHD) is a problem in the structure of the heart that is present at birth, with symptoms that can vary from none to life-threatening. ${ }^{1}$ CHD is the most
Strengths and limitations of this study

- We conducted a cross-sectional survey with high-quality face-to-face interviews.

- Multiple logistic regression was used to examine factors associated with parents' willingness to donate their children's biospecimens.

- All the participants were asked to rate their understanding of the questionnaire.

- We assessed parents' hypothetical willingness to donate biospecimens and their actual behaviour may differ.

- Our study was conducted at a single centre which limits the generalisability of our findings.

common congenital organ malformation in neonates, accounting for $28 \%$ of all malformations. $^{23}$

A number of genetic conditions are associated with heart defects, including Down syndrome, Turner syndrome and Marfan syndrome. Epidemiological studies strongly suggest genetic factors are the predominant cause of CHD, especially among young patients who need cardiopulmonary bypass surgery, although environmental exposure is also relevant. ${ }^{4}$ In combination with related clinical data, biospecimens (blood and residual tissue samples) are important materials for studying the aetiology of CHD. ${ }^{56}$

In general, for children to be involved in research in China, informed consent is provided by parents or legal guardians on behalf of their children. Among parents of children suffering from CHD who attended a cardiothoracic surgery inpatient department, the present study aimed to examine general willingness to donate their children's biospecimens to be frozen and stored in a biobank for future CHD research purposes. We also evaluated parents' attitudes and motivations regarding donating their children's biospecimens, their opinions about 
contributing biospecimens and ethical and legal considerations concerning their children's future willingness to donate biospecimens. In addition, we investigated parents' attitudes towards research results drawing on these biospecimens.

\section{STUDY DESIGN}

We conducted a cross-sectional survey of attitudes and perceptions regarding donating children's biospecimens for medical research among parents whose children suffered from CHD. This study was conducted in the cardiothoracic surgery inpatient department at Shanghai Children's Medical Center, one of the top CHD diagnosis and treatment centre in China (according to the Chinese hospital ranking from the Hospital Management Institute, Fudan University, http://rank.cn-healthcare. $\mathrm{com} / \mathrm{rank}$ /profession-reputation-best/24/2016), from 1 March 2016 to 31 December 2016. Inclusion criteria for participation were (1) having a direct relationship with the child; (2) being able to speak and read Mandarin Chinese; (3) being aged at least 20 years (women) or 22 years (men), because China's marriage law sets the legal age for marriage at 22 years for men and 20 years for women. This study was approved. All participants provided written informed consent.

Eligible participants were invited to attend face-toface interviews in a private interview room to complete a study questionnaire. The interviews were conducted by four interviewers (Shanghai Jiaotong University School of Medicine graduates), who received comprehensive training by the investigators about the study objective, participation criteria, questionnaire details, data collection, communication skills and protection of participants' information. The interviewers were instructed to explain each item and ensure that participants had the opportunity to ask questions and that there was sufficient time to think about their answers.

\section{Study questionnaire}

The questionnaire was designed with reference to previous related studies ${ }^{7-9}$ and focused on parents' demographic information and willingness to donate their children's biospecimens for medical research. Three reviewers independently evaluated the questionnaire and reached consensus on the items to be investigated. The questionnaire was tested in a pilot survey with 30 parents to ensure the participants understood the survey items and could provide clear answers. After revision and improvement, the final questionnaire covered gender, age, nationality (according to the official database, Han or national minority), residence (according to the official database, urban/Shanghai, rural/Shanghai, urban/other provinces or rural/other provinces), religion (Buddhism, Christianity, Islam or other), education (less than high school, high school, or college or graduate degree), children's history of hospitalisation with cardiac surgery, family history of CHD, only child or not and insurance status (national health insurance or private insurance). These variables were used to analyse the relationships between sociodemographic factors and parents' willingness to donate biospecimens.

Participants who were willing to donate biospecimens were also asked about their main motivation to donate biospecimens and their interest in acquiring research results relating to their children's biospecimens. To investigate motivations for donating biospecimens, participants were asked to select at least one of the following six options: (1) benefit for their children's future treatment; (2) helping other children with CHD; (3) inability to refuse the doctor's request; (4) benefit to their family; (5) making a contribution to medical developments and (6) other, which they were asked to explain briefly. Participants who were unwilling to donate biospecimens were asked to select at least one of the following six response options: (1) no self-benefit; (2) potential to cause physical discomfort to their children; (3) do not believe in researchers or the hospital; (4) worry about personal privacy being compromised; (5) worry about researchers receiving benefits from the biospecimens and (6) other, which they were asked to explain briefly.

All participants were asked if children aged $10-18$ years should be able to sign the informed consent form independently or if children should be asked to provide their own consent when they reach 18 years of age. Furthermore, participants who were willing to donate biospecimens were asked about whether they were interested in being informed of the research results regarding their children's donated biospecimens. At the end of the interview, all participants were asked to rate their understanding of the questions on a four-point Likert scale ranging from 'I understood less than half' to 'I understand everything'.

\section{Data analysis}

Participants' characteristics and responses were summarised as frequency counts and percentages. Associations between willingness to donate biospecimens and demographic and other factors were analysed using $\chi^{2}$ tests. Univariate logistic regression was used to examine the associations between willingness to participate in biospecimen research and sociodemographic and other factors. Variables identified as statistically significant at the 0.05 level in the univariate analyses were included in the multivariate analysis. Multiple logistic regression was used to examine whether demographic and other factors were associated with parents' willingness to donate biospecimens. ORs and 95\% CI were reported. The possible responses were 'agree', 'neutral' and 'disagree'. Responses of 'agree' were coded as 1, and responses of 'disagree' or 'neutral' (between agreement and disagreement) were coded as 0 ; thus, the ORs can be interpreted as the odds of consent. All tests were two-tailed, and $\mathrm{p}<0.05$ was considered statistically significant. All analyses were performed with SPSS, V.22.0 (SPSS Chicago, Illinois, USA). 


\section{Patient and public involvement}

Participants were not involved in the development of the research question or the design of this study. Eligible participants were recruited by the investigators in a private interview room. Participants were not involved in the recruitment or the conducting of the study. Dissemination of the general results (without personal data) will be made on request.

\section{RESULTS}

\section{Demographic characteristics}

In total, 550 parents (including the 30 parents who participated in the pilot survey) were invited to complete the questionnaire through a face-to-face interview; 42 declined to participate in the survey, yielding a response rate of 92.4\% (508/550). Participants' demographic characteristics are summarised in table 1 . The study population comprised $56.9 \%$ men and $43.1 \%$ women. The median age was 30 years (range: $21-49$ years), and approximately $97.0 \%$ of the participants were of Han nationality $(3.0 \%$ belonged to minority nationalities). Only $12.4 \%$ of the participants lived in Shanghai (7.7\% urban, $4.7 \%$ rural); the remaining $87.6 \%$ were from other provinces in China (35.2\% urban, $52.4 \%$ rural). In addition, $78.0 \%$ of the participants had no religion, and $35.6 \%$ had earned a college or graduate degree. More than half of the participants' children $(64.0 \%)$ had a history of hospitalisation, but only $5.7 \%$ had a family history of CHD. Almost half $(49.0 \%)$ of the sample had only one child; $51.0 \%$ had two or more children. Over half $(56.7 \%)$ of the participants had national health insurance.

\section{Factors associated with willingness to donate biospecimens for medical research}

In total, $69.1 \%(\mathrm{n}=351)$ of the participants were willing to donate their children's biospecimens and related clinical data for medical research, 20.1\% ( $\mathrm{n}=102)$ disagreed with donating and $10.8 \%(\mathrm{n}=55)$ reported a neutral attitude (between 'agree' and 'disagree') (table 2). A $\chi^{2}$ test showed that willingness to donate their children's biospecimens was significantly associated with education level and the child's history of hospitalisation $(\mathrm{p}<0.05)$. The multivariate analysis confirmed the association between willingness to donate and both education level (college or graduate degree: OR 2.435, 95\% CI 1.221 to 4.857, $\mathrm{p}=0.012$; high school: OR $1.827,95 \%$ CI 1.190 to 2.804, $\mathrm{p}=0.006)$ and history of hospitalisation (OR $1.581 ; 95 \% \mathrm{CI}$ 1.069 to $2.338, \mathrm{p}=0.022$ ) (table 3 ). The level of understanding of the questions in the questionnaire was not directly associated with willingness (table 2). Participants with higher education levels and those whose children had a previous history of hospitalisation were more likely to be willing to donate their children's biospecimens.

\section{Motivation for willingness/unwillingness to donate biospecimens}

Among the participants who were willing to donate their children's biospecimens $(\mathrm{n}=351), 81.2 \%(\mathrm{n}=285)$ thought
Table 1 Demographic characteristics of research participants, $\mathrm{n}(\%)$

\begin{tabular}{|c|c|}
\hline Characteristic & Total $(n=508)$ \\
\hline \multicolumn{2}{|l|}{ Gender } \\
\hline Male & 289 (56.9) \\
\hline Female & $219(43.1)$ \\
\hline \multicolumn{2}{|l|}{ Age (years) } \\
\hline $21-30$ & $277(54.5)$ \\
\hline $31-40$ & $187(36.8)$ \\
\hline$\geq 41$ & $44(8.7)$ \\
\hline \multicolumn{2}{|l|}{ Nation } \\
\hline Han & $493(97.0)$ \\
\hline National minority & $15(3.0)$ \\
\hline \multicolumn{2}{|l|}{ Residence } \\
\hline Urban of Shanghai & $39(7.7)$ \\
\hline Rural of Shanghai & $24(4.7)$ \\
\hline Urban of other provinces & $179(35.2)$ \\
\hline Rural of other provinces & $266(52.4)$ \\
\hline \multicolumn{2}{|l|}{ Religion } \\
\hline Yes & $112(22.0)$ \\
\hline No & $396(78.0)$ \\
\hline \multicolumn{2}{|l|}{ Education } \\
\hline Less than high school & $45(8.9)$ \\
\hline High school & $282(55.5)$ \\
\hline College or graduate degree & $181(35.6)$ \\
\hline \multicolumn{2}{|l|}{ History of hospitalisation } \\
\hline Yes & $325(64.0)$ \\
\hline No & $183(36.0)$ \\
\hline \multicolumn{2}{|l|}{ Family history } \\
\hline Yes & $29(5.7)$ \\
\hline No & 479 (94.3) \\
\hline \multicolumn{2}{|l|}{ Only child } \\
\hline Yes & $249(49.0)$ \\
\hline No & $259(51.0)$ \\
\hline \multicolumn{2}{|l|}{ Insurance } \\
\hline Yes & $288(56.7)$ \\
\hline No & $220(43.3)$ \\
\hline
\end{tabular}

doing so might help other children with CHD, 71.5\% $(\mathrm{n}=251)$ thought the donation would benefit their children's future treatment and $57.3 \%(\mathrm{n}=201)$ believed the donation would contribute to medical developments. Of the 102 participants who were unwilling to donate their children's biospecimens, $52.3 \% \quad(n=54)$ thought doing so might cause physical discomfort to their children, $47.1 \%$ $(\mathrm{n}=48)$ worried their personal privacy might be compromised and $27.5 \%(n=28)$ worried about researchers benefiting from the biospecimens. 
Open access

Table 2 Participants' willingness to donate their children's biospecimens, $\mathrm{n}(\%)$

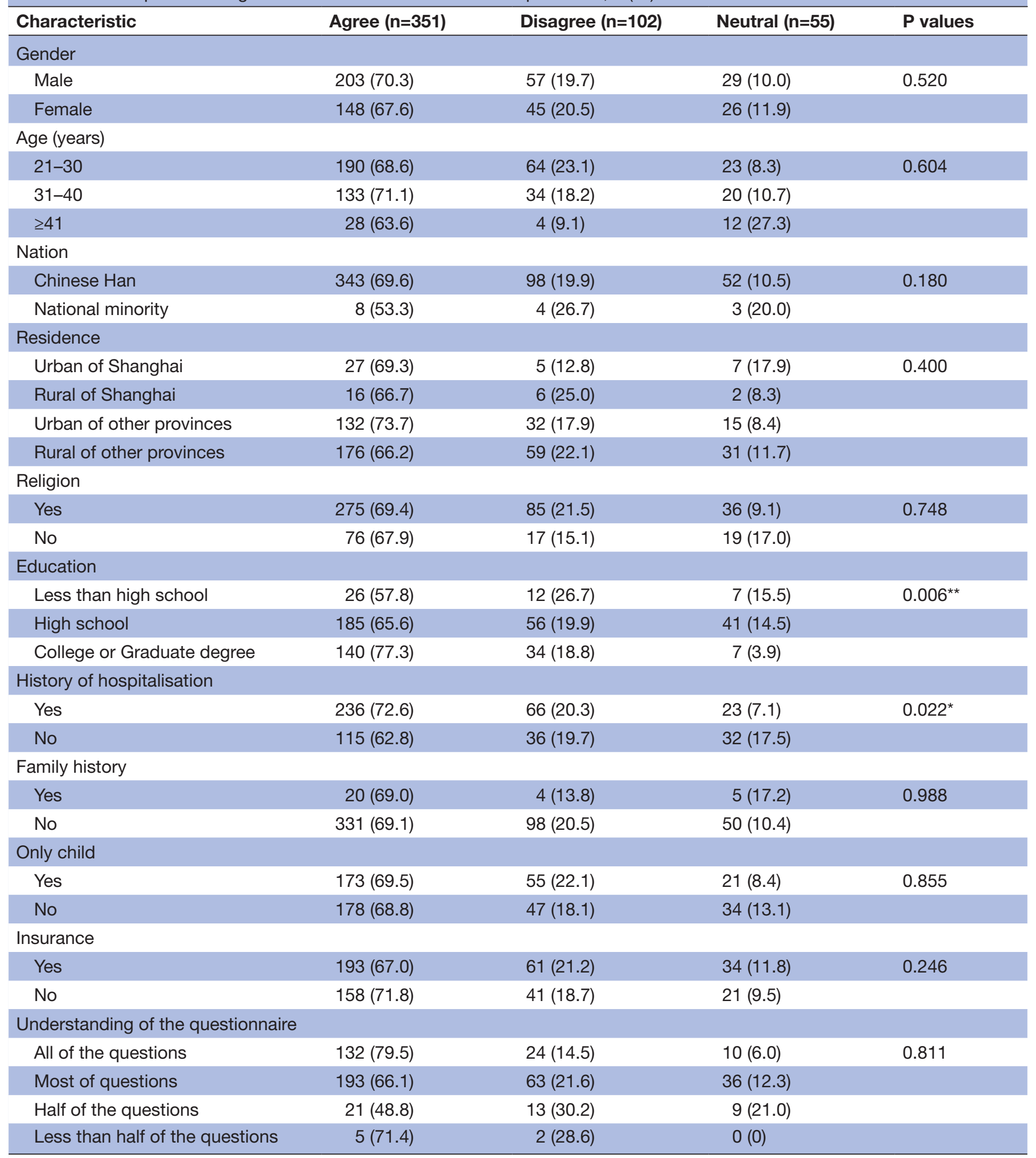

${ }^{*} \mathrm{p}<0.05,{ }^{* *} \mathrm{p}<0.01$.

Ethical issues about children's consent for research

In total, $34.8 \%(\mathrm{n}=177)$ of the participants believed that children aged 10-18 years had the right to consent independently to research participation, whereas the opposite opinion was held by $57.7 \% \quad(\mathrm{n}=293)$ of the participants. Furthermore, $75.4 \% \quad(\mathrm{n}=383)$ of the participants felt that children should provide their own consent for research when they grew older and reached a certain level of maturity.

Participants' attitudes towards biospecimen research results Of the participants who were willing to donate their children's biospecimens $(n=351), 86.0 \% \quad(n=302)$ wanted to 


\begin{tabular}{|c|c|c|}
\hline Characteristic & $P$ values & OR $(95 \% \mathrm{Cl})$ \\
\hline \multicolumn{3}{|l|}{ Education } \\
\hline $\begin{array}{l}\text { College or Graduate } \\
\text { degree }\end{array}$ & $0.012^{*}$ & 2.435 (1.221 to 4.857$)$ \\
\hline High school & $0.006^{\star \star}$ & 1.827 (1.190 to 2.804$)$ \\
\hline Less than high school & Reference & \\
\hline \multicolumn{3}{|l|}{ History of hospitalisation } \\
\hline Yes & $0.022^{*}$ & 1.581 (1.069 to 2.338 ) \\
\hline No & Reference & \\
\hline
\end{tabular}

${ }^{*} \mathrm{p}<0.05,{ }^{* *} \mathrm{p}<0.01$.

be informed about the research results involving their children's donated biospecimens, $10.3 \% \quad(\mathrm{n}=36)$ were indifferent about receiving the results and $3.7 \% \quad(n=13)$ indicated they did not wish to receive the results.

\section{Understanding of the questionnaire}

Around one-third $(32.7 \%, \mathrm{n}=166)$ of the participants indicated that they definitely understood all of the questions in the questionnaire, $57.5 \%(\mathrm{n}=292)$ understood most (more than two-thirds) of the questions, $8.4 \% \quad(n=43)$ understood half of the questions and $1.4 \%(n=7)$ understood fewer than half of the questions.

\section{DISCUSSION}

High-quality biospecimens and related clinical data are crucial for CHD research. Understanding the reasons underlying parents' attitudes towards donating their children's biospecimens for research purposes will improve the consent process and provide opportunities to resolve barriers and increase parents' willingness to donate biospecimens. We conducted a cross-sectional study among parents of children at a CHD centre to explore parental attitudes regarding the donation of biospecimens. Parents who were willing to donate their children's biospecimens and related clinical data made up approximately $69.1 \%$ of the sample, a percentage lower than those previously found for patients with cancer $(87.1 \%$ for leftover tissue, $83.3 \%$ for surplus blood after diagnosis). ${ }^{10}$ In 2012, Brothers et al reported that $88.6 \%$ of parents were willing to donate their child's biospecimens to a biobank in a paediatric hospital setting. ${ }^{11}$

Earlier studies confirmed that many factors (eg, gender, age, residence, religion, education and other demographic factors) are associated with participants' willingness to donate biospecimens for research. ${ }^{12-14}$ The present study has shown that parents' willingness to donate biospecimens was significantly associated with their education level and their children's history of hospitalisation. Specifically, parents with higher levels of education (college/graduate degree or high school) and those whose children had experienced hospitalisation were more willing to donate biospecimens for research.
Since the enactment of China's one-child family planning policy in the 1970s, a large number of couples (especially in urban areas) have had only one child; this means they focus their attention and protection on their only child. We hypothesised that parents with only one child would be less likely to donate their children's biospecimens. However, number of children did not affect parents' decisions regarding the donation of their children's biospecimens in this study. People are not likely to accept new ideas, especially with regard to unfamiliar topics. A previous study conducted by Frazier showed that older adults acknowledged their lack of understanding of genetic concepts, which resulted in their non-participation in genetic research studies. ${ }^{15}$ This is consistent with our finding that parents with a lower education level were more likely to refuse to donate their children's biospecimens. We also found that children's history of hospitalisation increased parents' willingness to donate biospecimens for CHD research. Experience with a child suffering from CHD may mean that these parents are more sensitive about and more willing to support research and have a stronger motivation to be involved in CHD research.

Participants in most previous studies stated that they might consider donating biospecimens if there was a direct benefit to themselves or their family members. ${ }^{716}$ Indeed, this was a key reason given by parents for their favourable views about the intrinsic merits of scientific research and donating biospecimens. Interestingly, the most common motivation for donating biospecimens found in our study was the potential benefit to other children. Rapid improvement in prenatal and postnatal early diagnosis as well as advances in interventional, surgical and postoperative treatments mean the survival of patients with CHD has increased dramatically in the last two decades. ${ }^{61718}$ After experiencing the difficulties of a child being diagnosed with CHD, having an operation and receiving postoperative treatment, parents were more likely to understand the experiences of other parents and sympathise with other children with CHD; therefore, they were more willing to donate their own children's biospecimens.

The main barrier to biospecimen donation was related to drawing blood or other intrusive methods, which might cause their children discomfort during the donation process. In addition, patients' mistrust of doctors is relatively common in China, ${ }^{10}$ as are concerns about personal privacy being compromised; however, a survey conducted by University of Chicago researchers reported that $87 \%$ of women agreed that researchers would keep their samples and medical information private. ${ }^{19}$ Improving public trust and confidence is necessary to ensure the long-term viability of biospecimen research.

Several sets of guidelines and the ethics literature note that children should be informed about what happens with their samples and the possibility of withdrawing them from a biobank as soon as they are mentally capable of understanding. ${ }^{20}$ Only one-third of our participants 
understood this principle, indicating that parents' understanding about ethical issues related to their children needs to be improved. About three-quarters of the participants in our study thought it was necessary for children to provide their own consent for biospecimen donation when they reach adulthood because of children's rights. However, obtaining this kind of later consent may not be feasible in all cases, operationally and financially. ${ }^{21} \mathrm{~A}$ previous qualitative focus group study suggested that a 'best effort' to recontact young adults regarding consent is sufficient and seen as a sign of respect from researchers. ${ }^{22}$

Most parents in our study wanted to receive the research results related to their children's biospecimens-especially the genetic research results. This finding has been confirmed in other surveys, where the return of research results was regarded as an influential factor in participants' decisions to become biobank donors, ${ }^{23}{ }^{24}$ with researchers endorsing the obligation of communicating with research participants.

\section{Strengths and limitations}

The strengths of this study included the high-quality face-to-face interviews and the protection of participants' privacy during the survey process. To our knowledge, this was the first such survey related to children with CHD. All interviews for this study were conducted in a separate room, and participants were given sufficient time to think about their answers. However, this study also had several limitations. First, we only surveyed participants' willingness to donate biospecimens, which might not translate into actual behaviour. Previous studies have verified that there are differences between willingness to consent and actually undergoing a research biopsy. ${ }^{14}{ }^{24}$ Second, $9.8 \%$ of the participants had difficulty understanding the questions. This was especially the case among parents with lower education levels. This difficulty might have affected the accuracy of the results, and more efforts should have been taken to explain each question clearly to the participants. Third, we did not ask all of the participants about both motivations and barriers. Fourth, parents who completed the questionnaire might have had more positive attitudes towards biospecimen research and greater willingness to donate biospecimens for research purposes, compared with the non-participating parents.

\section{CONCLUSIONS}

This study provides novel empirical data regarding the likelihood of donating biospecimens among parents of children with CHD in Shanghai, China. The overall results revealed that nearly $70 \%$ of these parents were willing to donate their children's biospecimens to be frozen and stored for future research. Parents with higher education levels and those whose children had a previous history of hospitalisation were more likely to be willing to donate biospecimens. Parents' most important motivation for donating biospecimens was that doing so might help other children who suffer from similar diseases, whereas potential physical discomfort for their children negatively influenced their willingness to donate. The majority of the parents in this study wanted to receive the research results related to their children's biospecimens. Future multicentre research and comparable studies of parents are needed to improve understanding of the factors associated with donating children's biospecimens for medical research.

Acknowledgements We gratefully acknowledge the valuable contribution of patient advisers, patients and their family members. We thank Audrey Holmes, MA, and Jennifer Barrett, PhD, from Liwen Bianji, Edanz Group China (www.liwenbianji. $\mathrm{cn} / \mathrm{ac})$, for editing the English text of a draft of this manuscript.

Contributors SL and HH designed the research. HG, HH, JJ, SL, BF and AG performed the study. $\mathrm{HG}$ and $\mathrm{SL}$ drafted the manuscript and performed the statistical analyses. SL and HH contributed to the interpretation of the results and critically reviewed the manuscript. SL had the primary responsibility for the final content. All authors read and approved the final manuscript and agreed to be accountable for all aspects of the work. No financial disclosures were reported by the authors of this article.

Funding This work was supported by Shanghai Collaborative Innovation Center for Translational Medicine grant number TM201720, Education and Teaching Reform Program for Graduate in Shanghai Jiao Tong University grant number YJG160207, Program of National Science and Technology Commission for the Association of Diabetes and Nutrition in Adolescents grant number 2016YFC1305203, Shanghai Children's Health Service Capacity Construction grant number GDEK201708, National Human Genetic Resources Sharing Service Platform grant number 2005DKA21300, Science and Technology Development Program of Pudong Shanghai New District grant number PKJ2017-Y01, Shanghai Jiaotong University School of Medicine Science Innovation Funding grant number Z2016-02 and ShanghaiProfessional and Technical Services Platform grant number $18 D Z 2294100$.

Competing interests None declared.

Patient consent Guardian consent obtained.

Ethics approval The study was approved by the institutional review boards of Shanghai Children's Medical Center.

Provenance and peer review Not commissioned; externally peer reviewed. Data sharing statement No additional data are available.

Open access This is an open access article distributed in accordance with the Creative Commons Attribution Non Commercial (CC BY-NC 4.0) license, which permits others to distribute, remix, adapt, build upon this work non-commercially, and license their derivative works on different terms, provided the original work is properly cited, appropriate credit is given, any changes made indicated, and the use is non-commercial. See: http://creativecommons.org/licenses/by-nc/4.0/.

\section{REFERENCES}

1. Majnemer A, Limperopoulos C, Shevell MI, et al. A new look at outcomes of infants with congenital heart disease. Pediatr Neurol 2009;40:197-204.

2. Dolk H, Loane M, Garne E, et al. Congenital heart defects in Europe: prevalence and perinatal mortality, 2000 to 2005 . Circulation 2011;123:841-9.

3. Hoffman JI, Kaplan S. The incidence of congenital heart disease. $J$ Am Coll Cardiol 2002;39:1890-900.

4. Gelb BD, Chung WK. Complex genetics and the etiology of human congenital heart disease. Cold Spring Harb Perspect Med 2014;4:a013953.

5. Opotowsky AR, Loukas B, Ellervik C, et al. Design and Implementation of a Prospective Adult Congenital Heart Disease Biobank. World J Pediatr Congenit Heart Surg 2016;7:734-43.

6. Pickardt T, Niggemeyer E, Bauer UM, et al. A Biobank for Long-term and Sustainable Research in the Field of Congenital Heart Disease in Germany. Genomics Proteomics Bioinformatics 2016;14:181-90.

7. Tong EK, Fung LC, Stewart SL, et al. Impact of a biospecimen collection seminar on willingness to donate biospecimens among Chinese Americans: results from a randomized, controlled community-based trial. Cancer Epidemiol Biomarkers Prev 2014;23:392-401. 
8. Kerath SM, Klein G, Kern M, et al. Beliefs and attitudes towards participating in genetic research - a population based cross-sectional study. BMC Public Health 2013;13:114.

9. Ahram M, Othman A, Shahrouri M, et al. Factors influencing public participation in biobanking. Eur J Hum Genet 2014;22:445-51.

10. He N, Guo Y, He M, et al. Attitudes and perceptions of cancer patients toward biospecimen donation for cancer research: A crosssectional survey among chinese cancer patients. Biopreserv Biobank 2017;15:366-74.

11. Brothers KB, Clayton EW. Parental perspectives on a pediatric human non-subjects biobank. AJOB Prim Res 2012;3:21-9.

12. Ma $\mathrm{Y}$, Kong $\mathrm{X}$, Dai $\mathrm{H}$, et al. Attitudes towards biosample donation in andrology patients. Int J Androl 2012;35:170-5.

13. Porteri C, Pasqualetti $P$, Togni E, et al. Public's attitudes on participation in a biobank for research: an Italian survey. BMC Med Ethics 2014;15:81.

14. Lee $\mathrm{Cl}$, Bassett LW, Leng M, et al. Patients' willingness to participate in a breast cancer biobank at screening mammogram. Breast Cancer Res Treat 2012;136:899-906.

15. Sanner JE, Frazier L. Factors that influence characteristics of genetic biobanks. J Nurs Scholarsh 2007;39:25-9.

16. Williams AM, Allen J, Zeps N, et al. Consent to donate surgical biospecimens for research: Perceptions of people with colorectal cancer. Cancer Nurs 2016;39:221-7.
17. Miranović $\mathrm{V}$. The incidence of congenital heart disease: previous findings and perspectives. Srp Arh Celok Lek 2014;142(3-4):243-8.

18. Sable $\mathrm{C}$, Foster $\mathrm{E}$, Uzark $\mathrm{K}$, et al. Best practices in managing transition to adulthood for adolescents with congenital heart disease: the transition process and medical and psychosocial issues: a scientific statement from the American Heart Association. Circulation 2011;123:1454-85.

19. Neidich AB, Joseph JW, Ober C, et al. Empirical data about women's attitudes towards a hypothetical pediatric biobank. Am J Med Genet A 2008;146A:297-304.

20. Kong CC, Tarling TE, Strahlendorf C, et al. Opinions of Adolescents and Parents About Pediatric Biobanking. J Adolesc Health 2016:58:474-80.

21. Klima J, Fitzgerald-Butt SM, Kelleher KJ, et al. Understanding of informed consent by parents of children enrolled in a genetic biobank. Genet Med 2014;16:141-8.

22. Rush A, Battisti R, Barton B, et al. Opinions of Young Adults on ReConsenting for Biobanking. J Pediatr 2015;167:925-30.

23. Murphy J, Scott J, Kaufman D, et al. Public expectations for return of results from large-cohort genetic research. Am J Bioeth 2008;8:36-43.

24. Gao W, Ma GX, Tan Y, et al. Factors associated with willingness to participate in biospecimen research among Chinese Americans. Biopreserv Biobank 2014;12:131-8. 